\title{
INTRAUTERINE GROWTH RESTRICTION: POSSIBLE INFLUENCE ON CORD BLOOD MANNOSE BINDING PROTEIN CONCENTRATIONS AT TERM
}

\author{
S. Liosi ${ }^{1}$, D.D. Briana ${ }^{1}$, M. Boutsikou ${ }^{1}$, A. Marmarinos ${ }^{2}$, T. Boutsikou ${ }^{1}$, S. Baka ${ }^{1}$, D. Hassiakos ${ }^{1}$, D. \\ Gourgiotis $^{2}$, A. Malamitsi-Puchner ${ }^{1}$ \\ ${ }^{l}$ Neonatal Division 2nd Department of Obstetrics and Gynecology, ${ }^{2}$ Research Laboratories, 2 nd Department
of Pediatrics, Athens University Medical School, Athens, Greece
}

Background and aims: Mannose binding protein (MBP) is a calcium-dependent serum protein playing role in the innate immune response by activating the lectin pathway of the complement system or acting directly as an opsonin. According to recent studies, MBP was found to aggravate reperfusion injury, while the lectin pathway of complement activation in general increases injury after intestinal ischemia. This study aimed to determine cord blood MBP concentrations in intrauterine-growth-restricted (IUGR) pregnancies, (characterized by placental ischemia, activation of the innate immune system and increased risk for necrotizing enterocolitis) and appropriate-for-gestational-age (AGA) pregnancies. Furthermore to investigate possible correlations of MBP concentrations with several demographic parameters of infants at birth.

Methods: Serum MBP concentrations were determined by ELISA in 160 mixed arteriovenous cord blood samples from 50 IUGR and 110 AGA singleton full-term pregnancies.

Results: Cord blood concentrations of MBP were significantly decreased in IUGR cases as compared to AGA controls $(p=0.038)$. Furthermore, cord blood MBP concentrations did not depend on birth weight, customized centile, gender, gestational age, mode of delivery or maternal age.

Conclusions: The significant decrease of MBP concentrations in IUGR cases, compared to AGA ones, probably represents a protective mechanism by inhibiting excessive complement activation and consequent tissue injury following ischemia/reperfusion. 